“ (C) 2017 IEEE. Personal use of this material is permitted. Permission from IEEE must be obtained for all other uses, in any current or future media, including

reprinting/republishing this material for advertising or promotional purposes, creating new collective works, for resale or redistribution to servers or lists, or reuse of any copyrighted component of this work in other works." 


\title{
Vector Control for a Bearingless Induction Motor Based on Nonsingular Terminal Sliding Mode Structure
}

\author{
Zebin Yang ${ }^{1 *}$, Dan Zhang ${ }^{1}$, Xiaodong Sun ${ }^{2}$, Weiming Sun ${ }^{1}$, Jianguo $\mathrm{Zhu}^{3}$, and Youguang Guo ${ }^{3}$ \\ ${ }^{1}$ School of Electrical and Information Engineering, Jiangsu University, Zhenjiang 212013, China \\ ${ }^{2}$ Automotive Engineering Research Institute, Jiangsu University, Zhenjiang 212013, China \\ ${ }^{3}$ School of Electrical, Mechanical and Mechatronic Systems, University of Technology, Sydney, NSW 2007, Australia \\ Email: zbyang@ujs.edu.cn
}

\begin{abstract}
To improve the performance of the bearingless induction motor (BIM) under disturbances, a nonsingular fast terminal sliding mode control (NFTSMC) strategy is proposed. The sliding mode surface is designed as a combination of linear sliding mode and nonsingular terminal sliding mode. Besides, considering the power function of the state variables, which make the approaching speed correlate with the state variables, so as to improve convergence performance of the linear sliding mode and solve the singularity of terminal sliding mode. Meanwhile, current signal and radial force are extracted by the electromagnetic torque and the equation of levitation force. Therefore, the convergence speed of system can be accelerated during the whole process, which contributes to chattering-free operating. The simulation and experiment results indicate that the proposed method can not only track the given value of the speed and radial displacement quickly, but also improve the operation quality and enhance the system robustness.
\end{abstract}

Index Terms--Bearingless induction motor (BIM), convergence speed, chattering, nonsingular fast terminal sliding mode control (NFTSMC), robustness

\section{INTRODUCTION}

The bearingless induction motor(BIM) integrates functions and advantages of asynchronous motor and magnetic bearing as a whole, such as simple structure, uniform air gap, low cogging torque ripple, no friction and wear, no noise, no additional lubrication and long service life [1-2]. The traditional PID controller has been widely used in control system of motors. However, when the system suffers from parameters variation or external uncertain disturbance factors, the BIM with nonlinear, multivariate, strong coupling characteristics is hard to acquire excellent performance by using the traditional PID control [3].

Great deals of studies have been done by domestic and foreign scholars, certain of advanced control strategies have been gradually applied to the control system. Generally, these methods can be classified into four main strategies, involving adaptive control, fuzzy control, neural network control and sliding mode control [4-5] Sliding mode variable structure control (SMC) is gradually applied to the AC servo system because of its sliding trajectory can be set up automatically, without high accuracy requirement to the mathematical model and excellent robustness to the disturbance, SMC has been developed as one of effective methods [6-7]. In [8], a novel reaching law based on power function of system state variables and an extended disturbance observer is proposed, the dynamic performance of permanent magnet synchronous motor speed control system can be improved, which enhances the robustness of the system effectively. However, it is too complication for engineering applications. In [9], a robust fuzzy neural network slidingmode control is proposed. The results demonstrate that the dynamic behaviors of the proposed control systems are robust to the uncertainties. However, it is difficult to select index parameters and it limits the application of high precision motors. In [10], fault-tolerant control based on nonsingular terminal sliding-mode control and nonsingular fast terminal sliding-mode control is investigated. It has the advantages of fast response, easy implementation. In [11], a practical nonsingular terminal sliding-mode (TSM) tracking control design for robot manipulators using time-delay estimation (TDE) is presented. However, this method only considers fast convergence while ignoring the effects of the nonsingular terminal sliding mode when system state far away from the equilibrium point, so it is unable to achieve the optimum control performance.

In order to track the speed and radial displacement of BIM accurately, a nonsingular fast terminal sliding mode control (NFTSMC) strategy is investigated. This method not only avoids the disadvantage from the deficiency of linear sliding mode tends to the equilibrium point asymptotically, but also overcomes the shortcoming of the nonsingular terminal sliding mode slow convergence rate when the state of the system far away from the equilibrium point, so that the whole system has global rapid convergence. It is greatly significant to apply this method to design the speed controller and the radial displacement controller. Meanwhile, the smoothing of the symbol function can effectively suppress the inherent chattering of the sliding mode. The simulation and experimental results show that the proposed method can track the given value of the rotation speed and the radial displacement rapidly. Moreover, it has the characteristics of fast convergence and response, the system chattering of sliding mode is weakened simultaneously.

\section{PERFORMANCE ANALYSIS OF NFTSMC}

A nonlinear control system is given as: 


$$
\left\{\begin{array}{l}
\dot{x}_{1}=x_{2} \\
\dot{x}_{2}=f(x)+g(x)+b(x) u
\end{array}\right.
$$

where $x=\left[x_{1}, x_{2}\right]^{T}$ are variables of the system state. $b(x) \neq 0 . g(x)$ is the uncertain disturbances of system, $|g(x)| \leq \varsigma, \varsigma$ is the upper bound of disturbance.

By adopting the exponential reaching law to weaken the intrinsic chattering, it can be expressed as:

$$
\frac{d s}{d t}=-\xi \operatorname{sgn}(s)-\lambda s
$$

A nonsingular fast terminal sliding mode surface is proposed as:

$$
\begin{gathered}
\quad s=\alpha x_{1}+c x_{2}+\beta x_{2}{ }^{p / q} \\
\text { where } c=\left\{\begin{array}{l}
\varepsilon,|X| \geq 1 \\
\varepsilon|X|^{2},|X|<1
\end{array} .\right.
\end{gathered}
$$

When the system state variables are far away from the sliding mode surface, the system state variables are mainly close to the sliding mode surface through $\alpha x_{1}+c x_{2}$. The convergence speed can be accelerated. When the system state variables are close to the stable point, the $|X|$ gradually decreases and tends to zero, the coefficient decreases exponentially with the decrease of the state variables, $c x_{2}$ will tend to zero. Hence, the sliding mode dynamics will stabilize at the origin. The nonsingular terminal sliding mode $\alpha x_{1}+\beta x_{2}{ }^{p / q}$ will play an important role.

The controller can be designed as:

$$
\begin{aligned}
u= & -b^{-1}(x)\left[f(x)+\frac{\alpha}{c+\frac{\beta p}{q} x_{2}{ }^{p / q-1}} x_{2}\right. \\
& \left.+\left(l_{g}+\xi\right) \operatorname{sgn}(s)+\lambda s\right]
\end{aligned}
$$

where $p, q, \alpha, \beta, \varepsilon, \lambda, \xi$ are constants greater than zero; $1<p / q<2 ; X$ is the system state variable $x_{1}$ or $x_{2}$. As shown in the formula (6), $u$ will not appear the phenomenon of infinity, it can be seen that the system can converge to the stable point within a limited time.

According to the Lyapunov stability theory, the existence and reachability of sliding mode are expressed as:

$$
\dot{V}=s \dot{s}<0
$$

Substituting formula (1) (4) into (5), $p-q$ is known as even, $g(x) \leq l_{g}$. Then, Formula (5) turns into:

$$
\begin{aligned}
s \dot{s} & =s \cdot\left[c+\frac{\beta p}{q} x_{2}^{(p-q) / q}\right] \cdot\left[\frac{\alpha x_{2}}{c+\frac{\beta p}{q} x_{2}^{p / q-1}}+f(x)+g(x)+b(x) u\right] \\
& =s \cdot\left[c+\frac{\beta p}{q} x_{2}^{(p-q) / q}\right]\left[g(x)-\left(l_{g}+\xi\right) \operatorname{sgn}(s)-\lambda s\right] \\
& \leq\left[c+\frac{\beta p}{q} x_{2}^{(p-q) / q}\right] \cdot\left(-\xi|s|-\lambda s^{2}\right) \leq 0
\end{aligned}
$$

As a consequence, the NFTSMC satisfies the demands of the Lyapunov stability theory, the system can arrive at the sliding mode surface within a limited time from any state.
Taking representative nonlinear system: $\left\{\begin{array}{l}\dot{x}_{1}=x_{2} \\ \dot{x}_{2}=-25 x+0.1 \sin (20 t)+133 u\end{array}\right.$ as an example, the superiority of the proposed method can be verified by designing the nonsingular terminal sliding mode controller (NTSMC) and NFTSMC, respectively.

Choosing the initial value of the state variables from two aspects to analyze: $x(0)=[0.3,0.3]^{T}$ (the system state variables close to the equilibrium point) and $x(0)=[30,30]^{T}$ (the system state variables far away from the equilibrium point). The simulation results are shown in Fig. 1 and Fig. 2 , it can be seen that NFTSMC has faster convergence speed than NTSMC. What's more, the chattering is obviously suppressed.

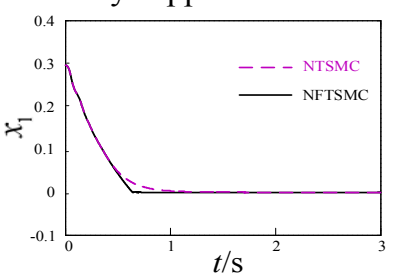

(a)

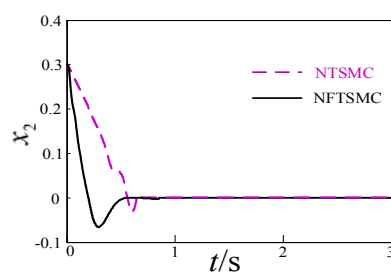

(b)

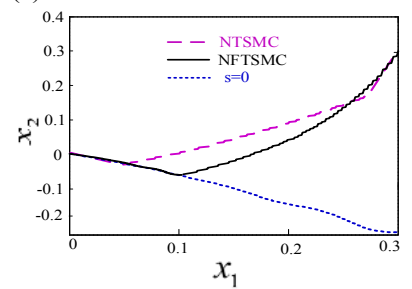

(c)

Fig.1. The performance comparison between NFTSMC and NTSMC. (a) The response of $x_{1}$. (b) The response of $x_{2}$. (c) The phase track of system.

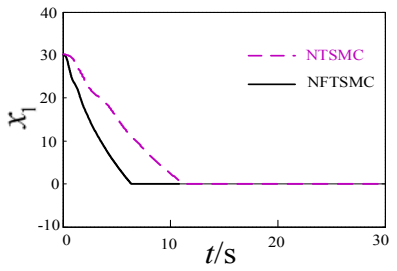

(a)

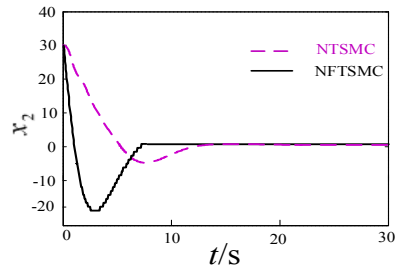

(b)

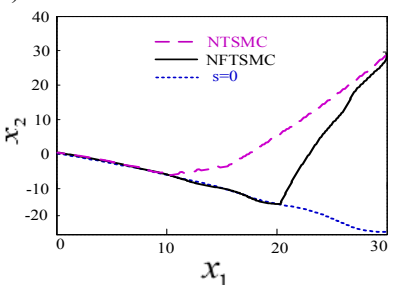

(c)

Fig.2. The performance comparison between NFTSMC and NTSMC. (a) The response of $x_{1}$. (b) The response of $x_{2}$. (c) The phase track of system.

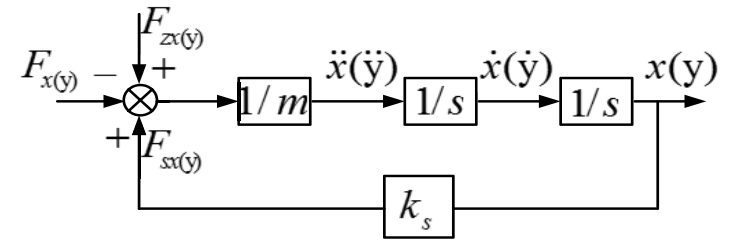

Fig.3. The diagram of motion equation of suspension rotor system. 


\section{DESIGN OF THE BIM RotATIONAL SPEED AND RADIAL DISPLACEMENT WITH NFTSMC}

\section{A. The Mathematical Model of BIM}

The equation of motion can be set up as:

$$
T_{e}-T_{l}-F \omega_{r}=\frac{J}{P_{1}} \frac{d \omega_{r}}{d t}
$$

where $\omega_{r}$ is the rotor speed; $J$ is the rotational inertia of rotor; $T_{l}$ is the load torque; $p$ is the differential operator; $F$ is the coefficient of friction between rotor and load.

Supposing the rotor mass of the motor is $m$, the $x$ and $y$ axis interference forces are individually as $F_{z x}$ and $F_{z y}$. The motion of BIM suspended rotor can be expressed as:

$$
\left\{\begin{array}{l}
F_{z x}+F_{s}-F_{x}=\ddot{m} \\
F_{z y}+F_{s y}-F_{y}=\ddot{m}
\end{array}\right.
$$

where $\left\{\begin{array}{l}F_{s x}=k_{s} x \\ F_{s y}=k_{s} y\end{array}\right.$.

where $F_{s}$ is the unbalanced magnetic force produced by the rotor eccentricity; $k_{s}$ is the radial force displacement stiffness, which is merely related to the structure of the motor. The motion equation of BIM suspension rotor system can be shown as Fig.3.

\section{B. The Design of the Speed with NFTSMC}

The system state variables can be defined as:

$$
\left\{\begin{array}{l}
e_{\omega 1}=\omega^{*}-\omega \\
e_{\omega 2}=\dot{e}_{\omega 1}=-\dot{\omega}
\end{array}\right.
$$

where $\omega^{*}$ is the given speed, $\omega$ is the actual speed.

From formula (7), (9), $\dot{e}_{\omega 2}$ can be derived as:

$$
\dot{e}_{\omega 2}=\ddot{e}_{\omega 1}=-\frac{p_{1}^{2} \psi_{1}}{J} \dot{i}_{q s}+\frac{F}{J} \dot{\omega}
$$

Because of the uncertain disturbances in the motor system, $\dot{e}_{\omega 2}$ can be expressed as:

$$
\dot{e}_{\omega 2}=\left(-\frac{p_{1}^{2} \psi_{1}}{J}+\Delta \zeta_{1}\right) \dot{i}_{q s}+\left(\frac{F}{J}+\Delta \eta_{1}\right) \dot{\omega}+\Delta \xi_{1}
$$

where $\Delta \zeta_{1}, \Delta \xi_{1}$ and $\Delta \eta_{1}$ are corresponding to the uncertain disturbances and bounded constants. Generally, $g_{1}(t)$ can be recorded as the total interference factors of the system, thus, $\dot{e}_{\omega 2}$ can be simplified as:

$$
\dot{e}_{\omega 2}=-\frac{p_{1}^{2} \psi_{1}}{J} \dot{i}_{q s}+\frac{F}{J} \dot{\omega}+g_{1}(t)
$$

where $g_{1}(t)=\Delta \zeta_{1} \dot{i}_{q s}+\Delta \eta_{1} \dot{\omega}+\Delta \xi_{1},\left|g_{1}(t)\right| \leq l_{g 1}$ and $l_{g 1}$ is a bounded positive constant.

Therefore, the system state equation of speed error can be obtained as:

$$
\left\{\begin{array}{l}
\dot{e}_{\omega 1}=e_{\omega 2}=-\frac{p_{1}^{2} \psi_{1}}{J} i_{q s}+\frac{p_{1}}{J} T_{L}+\frac{F}{J} \omega \\
\dot{e}_{\omega 2}=\ddot{e}_{\omega 1}=-\frac{p_{1}^{2} \psi_{1}}{J} i_{q s}+\frac{F}{J} \dot{\omega}+g_{1}(\mathrm{t})
\end{array}\right.
$$

A nonsingular fast terminal sliding mode can be chosen as:

$$
s=\alpha_{1} e_{\omega_{1}}+c_{1} e_{\omega_{2}}+\beta_{1} e_{\omega_{2}}^{p_{1} / q_{1}}
$$

The controller can be designed as:

$$
\begin{aligned}
i_{q s}= & \frac{J}{p_{1}^{2} \psi_{1}} \int\left[-\frac{F}{J} e_{\omega 2}+\left(\alpha_{1} e_{\omega 2}\right) /\left(c_{1}+\frac{\beta_{1} p_{1}}{q_{1}} e_{\omega 2}^{p_{1} / q_{1}^{-1}}\right)\right. \\
& \left.+\left(l_{g 1}+\xi_{1}\right) \operatorname{sgn}\left(s_{1}\right)+\gamma_{1} s_{1}\right] d t
\end{aligned}
$$

It can be seen that the current by integrating filter, which can reduce the steady-state error and improve the control precision. Furthermore, the speed error can converge to the expected place within a limited time.

\section{Design of Rotor Radial Displacement with NFTSMC}

The system state variables can be defined as:

$$
\left\{\begin{array}{l}
e_{x 1}=x^{*}-x \\
e_{x 2}=\dot{e}_{x 1}=-\dot{x}
\end{array}\right.
$$

where $x^{*}$ is the given displacement, $x$ is the actual displacement.

From formula (8), (16), $\dot{e}_{x 2}$ can be derived as:

$$
\dot{e}_{x 2}=\ddot{e}_{x 1}=\frac{1}{m} F_{x}+\frac{k_{s}}{m} e_{x 1}-\frac{1}{m} F_{z x}-\frac{k_{s}}{m} x^{*}
$$

Similarly, because of the uncertain disturbances in the motor system, $\dot{e}_{x 2}$ can be expressed as:

$$
\dot{e}_{x 2}=\left(\frac{1}{m}+\Delta \zeta_{2}\right) F_{x}+\left(\frac{k_{s}}{m}+\Delta \eta_{2}\right) e_{x 1}+\left(\Delta \xi_{2}-\frac{1}{m} F_{z x}-\frac{k_{s}}{m} x^{*}\right)
$$

where $\Delta \zeta_{2}, \Delta \xi_{2}$ and $\Delta \eta_{2}$ are corresponding to the uncertain disturbances and bounded constants. Generally, $g_{2}(t)$ can be recorded as the total interference factors of the system, thus, $\dot{e}_{\omega 2}$ can be simplified as:

$$
\dot{e}_{x 2}=\frac{1}{m} F_{x}+\frac{k_{s}}{m} e_{x 1}+g_{2}(\mathrm{t})
$$

where $\quad g_{2}(t)=\Delta \zeta_{2} F_{x}+\Delta \eta_{2} e_{x 1}+\left(\Delta \xi_{2}-\frac{1}{m} F_{z x}-\frac{k_{s}}{m} x^{*}\right) \quad ，$ $\left|g_{2}(t)\right| \leq l_{g_{2}}$ and $l_{g_{2}}$ is a bounded positive constant.

Consequently, the system state equation of radial displacement error can be obtained as:

$$
\left\{\begin{array}{l}
\dot{e}_{x 1}=e_{x 2} \\
\dot{e}_{x 2}=\frac{1}{m} F_{x}+\frac{k_{s}}{m} e_{x 1}+g_{2}(\mathrm{t})
\end{array}\right.
$$

A nonsingular fast terminal sliding mode can be chosen as:

$$
s=\alpha_{2} e_{x_{1}}+c_{2} e_{x_{2}}+\beta_{2} e_{x_{2}}^{p_{2} / q_{2}}
$$

The controller $F_{x}$ can be designed as:

$$
\begin{aligned}
F_{x}= & -m\left\{\frac{k_{s}}{m} e_{x 1}+\left(\alpha_{2} e_{x 2}\right) /\left(c_{2}+\frac{\beta_{2} p_{2}}{q_{2}} e_{x 2}^{p_{2} / q_{2}^{-1}}\right)\right. \\
& \left.+\left(l_{g 2}+\xi_{2}\right) \operatorname{sgn}\left(s_{2}\right)+\gamma_{2} s_{2}\right\}
\end{aligned}
$$

Similarly, the $F_{y}$ controller can be designed as:

$$
\begin{aligned}
F_{y}= & -m\left\{\frac{k_{s}}{m} e_{y 1}+\left(\alpha_{2} e_{y 2}\right) /\left(c_{2}+\frac{\beta_{2} p_{2}}{q_{2}} e_{y 2}^{p_{2} / q_{2}}{ }^{-1}\right)\right. \\
& \left.+\left(l_{g 2}+\xi_{2}\right) \operatorname{sgn}\left(s_{2}\right)+\gamma_{2} s_{2}\right\}
\end{aligned}
$$

Therefore, the $x, y$ direction radial displacement error can converge to the expected place within a limited time. 


\section{SIMULATION AND EXPERIMENTAL VERIFICATION}

\section{A. Results and Analysis of Simulation}

The structure diagram of BIM control system based on NFTSMC is shown as Fig.4. Compared with the difference between the speed $\omega_{r}$ and the given speed $\omega_{r}^{*}$. Then, by means of NFTSMC controller, the torque $T_{e}{ }^{*}$ can be derived. In order to verify the validity of the proposed control method, Matlab/Simulink is adopted to build the simulation models of BIM control system. Table I gives the structure parameters of the BIM.

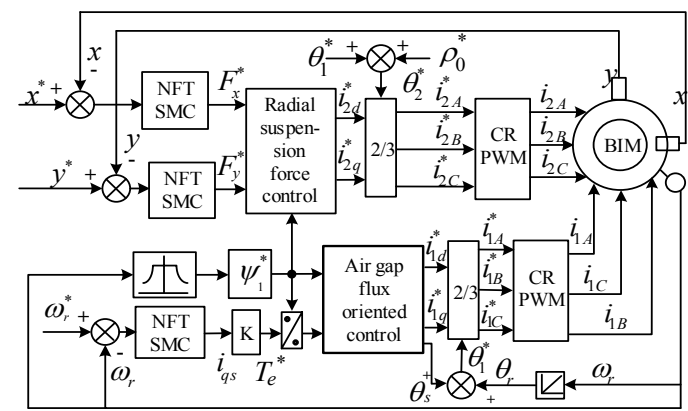

Fig.4. The structure diagram of NFTSMC system of BIM.

TABLE I

PARAMETERS OF THE BIM

\begin{tabular}{ccc}
\hline Parameters & Torque winding & $\begin{array}{c}\text { Suspension } \\
\text { winding }\end{array}$ \\
\hline Rated current $(\mathrm{A})$ & 2.86 & 2.86 \\
Stator resistance $(\Omega)$ & 2.01 & 1.03 \\
Rotor resistance $(\Omega)$ & 11.48 & 0.075 \\
Mutual inductance of stator & 0.15856 & 0.00932 \\
and rotor $(\mathrm{H})$ & 0.16310 & 0.01199 \\
Stator leakage inductance & & \\
$(\mathrm{H})$ & 0.16778 & 0.01474 \\
Rotor leakage inductance & 0.00769 & 0.00769 \\
$(\mathrm{H})$ & 2.85 & 2.85 \\
Rotational inertia $\left(\mathrm{kg} \bullet \mathrm{m}^{2}\right)$ & 98 & 98 \\
Rotor mass $(\mathrm{kg})$ & 105 & 105 \\
Stator inner diameter $(\mathrm{mm})$ & 0.0001 & - \\
Core length $(\mathrm{mm})$ & 1 & 2 \\
Friction coefficient & & \\
Pole pairs &
\end{tabular}

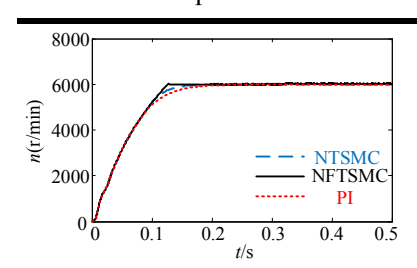

(a)

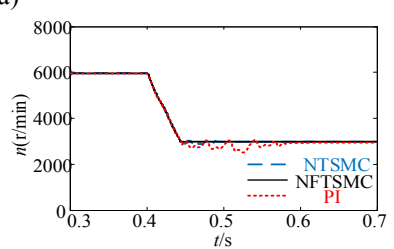

(c)

Fig.5. Waveforms of the speed dynamic response. (a) The speed response of no-load. (b) The speed response of the sudden load. (c) The response of speed mutation under the torque disturbance.

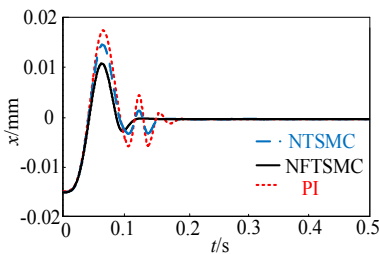

(a)

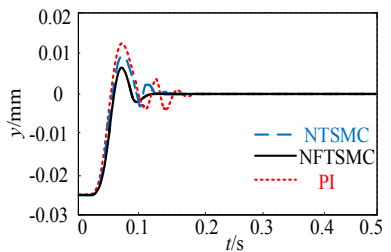

(b)
Fig.6. Waveforms of rotor radial displacement. (a) The radial displacement of $\mathrm{x}$-axis. (b) The radial displacement of $\mathrm{y}$-axis.

Fig. 5 shows the simulation results of the speed response. The parameters of NFTSMC are shown as : $p_{1}=9, q_{1}=7, \alpha_{1}=70, \varepsilon_{1}=1, \beta_{1}=0.1, \xi_{1}=0.3$, $\gamma_{1}=2, l_{g 1}=50$. The PI controller parameters are adjusted as: $k_{p}=1, k_{i}=0.001$. As shown in Fig. 5(a), the speed response of the system starts to $6000 \mathrm{r} / \mathrm{min}$ with noload, during the start-up process, compared with NFTSMC, the starting speed under the PI and NTSMC control is relatively slower, it can be found that the speed response of the system is fluctuated obviously and the overshoot is large, simultaneously. As shown in Fig. 5(b), the speed response of the system suffers from mutation load $(4 \mathrm{~N} \cdot \mathrm{m})$ when the system operating at the steady state, because of the affection of the external disturbances, the speed on the condition of the PI and NTSMC control is more sensitive to the load disturbance than NFTSMC, there are much longer time to reach the given speed. As shown in Fig. 5(c), the speed falls shapely from $6000 \mathrm{r} / \mathrm{min}$ to $3000 \mathrm{r} / \mathrm{min}$ under $4 \mathrm{~N} \cdot \mathrm{m}$ load, the speed fluctuates greatly and the dynamic regulation time is longer under the PI and NTSMC control in comparison with NFTSMC. Based on the above investigation, from the comparison of three selected methods, it has been verified that it is optimal to choose the NFTSMC control strategy, which contributes to the motor has the advantages of fast response, small overshoot, no fluctuation of the speed, and fast recovery with the given value.

Fig.6 shows the rotor radial displacement simulation results of the system operating at the speed mutation from 6000 to 3000 . The parameters of NFTSMC are shown as: $p_{2}=9, q_{2}=7, \alpha_{2}=1, \varepsilon_{2}=0.001, \beta_{2}=0.1, \xi_{2}=0.1$, $\gamma_{2}=0.5, l_{g 2}=20$. The PI controller parameters are adjusted as: $\mathrm{kp}=900, \mathrm{ki}=0.01$. The NFTSMC can approach the stable position quickly and suspend steadily, strong anti-disturbance ability, excellent robustness and better control performance can be obtained.

\section{B. Results and Analysis of the Experiment}

In order to further verify the superiority of the proposed method. The system experimental platform is built, which designed by laboratory of BIM is employed; the TMS320F2812 DSP is used in the experiment. Taking advantage of photoelectric encoder to measure the motor speed and the eddy current displacement sensor to detect the rotor radial displacement simultaneously, the prototype parameters are the same as simulation parameters, and setting the speed to $3000 \mathrm{r} / \mathrm{min}$, the air gap of the motor auxiliary bearing is $0.4 \mathrm{~mm}$, the hardware structure diagram is shown in Fig.7. 


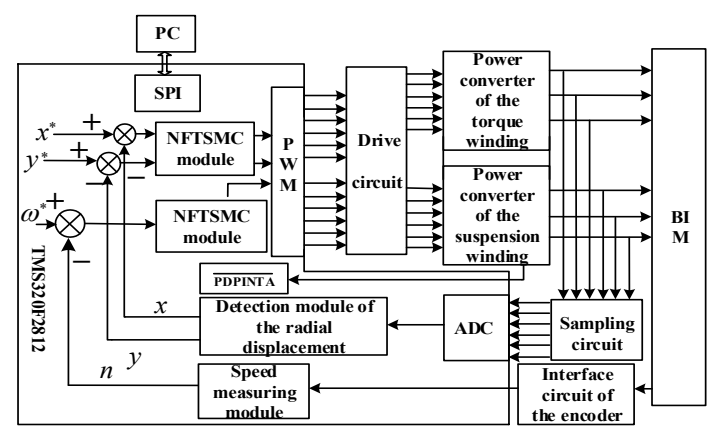

Fig.7. The experiment block diagram of the control system.

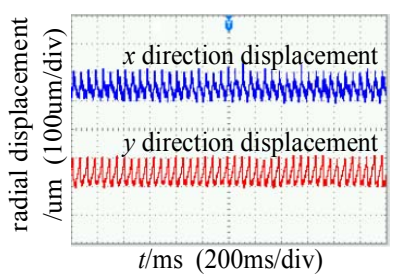

(a)

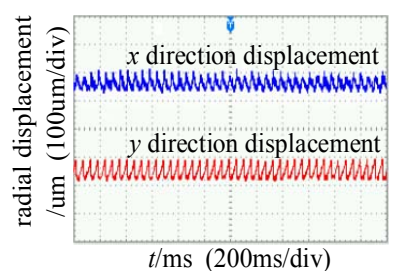

(b)

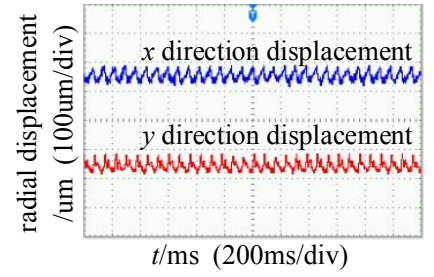

(c)

Fig.8. Experimental results of radial displacement. (a) The rotor radial displacement controlled by PI. (b) The rotor radial displacement controlled by NTSMC. (c) The rotor radial displacement controlled by NFTSMC.

As shown in Fig.8 (a), (b) and (c), the radial displacement curves of $x, y$ direction for the BIM operates stably at 3000r/min. From Fig.8 (a), (b), it can be seen that the vibration displacement under the control of PI and NTSMC, the peak to peak values are $100 \mu \mathrm{m}$ and $80 \mu \mathrm{m}$, respectively, which is far less than the length of the air gap of the motor. Therefore, the stable suspension can be realized. Fig.8(c) shows radial displacement experimental result under the control of NFTSMC, the vibration displacement of the peak to peak value is $60 \mu \mathrm{m}$. Compared with the PI and NTSMC, the NFTSMC amplitude are decreased by $40 \%$ and $20 \%$, respectively. Accordingly, it can be found that the suppression effect is obviously, the system control precision can be improved and the stable suspension of BIM can be ensured as well.

\section{CONCLUSION}

In order to quickly track the speed and the radial displacement of the BIM, a nonsingular fast terminal sliding mode control is proposed. It has been found that it is greatly important, by introducing the state variables, to improve the convergence speed and achieve the global fast convergence. Therefore, the proposed optimal method is effective and meaningful for improving the speed of response of BIM. The simulation and experimental results show that the speed controller and the radial displacement controller of BIM can not only improve the speed of system and restrain the chattering simultaneously, but also enhance the robustness of the system. In addition, it is effective to improve the dynamic and static performance of the BIM control system.

\section{ACKNOWLEDGMENT}

This work was supported by the National Natural Science Foundation of China (51475214 and 51305170), the Natural Science Foundation of Jiangsu Province of China (BK20141301 and BK20170071), the China Postdoctoral Science Foundation (2015T80508 and 2016M601726), the Six Categories Talent Peak of Jiangsu Province (2014-ZBZZ-017, 2015-XNYQC-003 and 2016-GDZB-096), Zhenjiang Key Research and Development Project (GY2016003), the "333 project" of Jiangsu Province (BRA2017441), and the Priority Academic Program Development of Jiangsu Higher Education Institutions (PAPD).

\section{REFERENCES}

[1] X. Sun, Z. Shi, L. Chen, and Z. Yang, "Internal model control for a bearingless permanent magnet synchronous motor based on inverse system method," IEEE Trans. Ener. Conv., vol. 31, no. 4, pp. 1539-1548, Dec. 2016.

[2] S. Zhang, L. Liu, S. Wang, Y. Jia and C. Qie, "Complete control of radial suspension force for bearingless induction motors," IEEE Proc. of 11th Conf. Ind. Electro. Appl. (ICIEA 2016), Hefei, 2016, pp. 2180-2184.

[3] L. Angel and J. Viola, "Design and statistical robustness analysis of FOPID, IOPID and SIMC PID controllers applied to a motor-generator system," IEEE Lat. Am. Trans., vol. 13, no. 12, pp. 3724-3734, Dec. 2015.

[4] R. Zhang, L. Dong and C. Sun, "Adaptive nonsingular terminal sliding mode control design for near space hypersonic vehicles," IEEE/CAA J. Autom. Sin., vol. 1, no. 2, pp. 155-161, Apr. 2014.

[5] Y. Wang, L. Gu, Y. Xu and X. Cao, "Practical tracking control of robot manipulators with continuous fractionalorder nonsingular terminal sliding mode," IEEE Trans. Ind. Electron., vol. 63, no. 10, pp. 6194-6204, Oct. 2016.

[6] H. Castañeda, F. Plestan, A. Chriette and J. de LeónMorales, "Continuous Differentiator Based on Adaptive Second-Order Sliding-Mode Control for a 3-DOF Helicopter," IEEE Trans. Ind. Electron., vol. 63, no. 9, pp. 5786-5793, Sept. 2016.

[7] Z. Yang, L. Wan, X. Sun, L. Chen and Z. Chen, "Sliding mode control for bearingless induction motor based on a novel load torque observer, "Journal of Sensors, 2016, pp. 1-10, Art. ID 8567429.

[8] X. Zhang, L. Sun, K. Zhao and L. Sun, "Nonlinear speed control for PMSM system using sliding-mode control and disturbance compensation techniques," IEEE Trans. Power Electron., vol. 28, no. 3, pp. 1358-1365, Mar. 2013.

[9] J. Lin and H. Shen, "Robust Fuzzy Neural Network Sliding-Mode Control for Two-Axis Motion Control System," IEEE Trans. Ind. Electron., vol. 53, no. 4, pp. 1209-1225, Jun. 2006.

[10] D. Xu, C. Chen and L. Wu, "Study of Nonsingular Fast Terminal Sliding-Mode Fault-Tolerant Control," IEEE Trans. Ind. Electron., vol. 62, no. 6, pp. 3906-3913, Jun. 2015.

[11] M. Jin, J. Lee, P. H. Chang and C. Choi, "Practical Nonsingular Terminal Sliding-Mode Control of Robot Manipulators for High-Accuracy Tracking Control," IEEE Trans. Ind. Electron., vol. 56, no. 9, pp. 3593-3601, Sept. 2009. 